\title{
Cytological and transcriptome analyses reveal abrupt gene expression for meiosis and saccharide metabolisms that associated with pollen abortion in autotetraploid rice
}

\author{
Lin Chen ${ }^{1,2} \cdot$ Muhammad Qasim Shahid ${ }^{1,2} \cdot$ Jinwen $\mathrm{Wu}^{2} \cdot$ Zhixiong Chen $^{2} \cdot$ Lan Wang ${ }^{2} \cdot$ Xiangdong Liu $^{1,2} \mathbb{D}$
}

Received: 7 January 2018 / Accepted: 1 July 2018 / Published online: 4 July 2018

(c) The Author(s) 2018

\begin{abstract}
Autotetraploid rice is a useful germplasm that has four chromosome sets and strong biological advantages; however, low fertility limits its commercial utilization. Little information is available about the DNA variation and differential gene expressions associated with low fertility in autotetraploid rice. In the present study, 81 SNPs and 182 InDels were identified in T449 (an autotetraploid rice line with low fertility) compared to E249 (diploid counterpart) by whole-genome re-sequencing. We detected only three non-synonymous SNPs and six large-effect InDels, which were associated with three and six genes, respectively. A total of 75 meiosis-related differentially expressed genes were detected during the meiosis stage by transcriptome analysis, including $O S M T O P V I B$, which is essential for meiotic DSB formation, and $O S M O F$, which takes part in homologous chromosome pairing and synapsis. Approximately $20.69 \%$ lagging chromosome at metaphase I and $4.65 \%$ abnormal tetrad were observed in T449. Moreover, transcriptome analysis revealed down-regulation of a sucrose transporter (OsSUT5) and two monosaccharide transporters (OSMST1 and OSMST8) in T449 at the single microspore stage, and their expression levels were verified by qRT-PCR. Cytological observation of saccharide distribution showed abnormal accumulation of saccharides in T449 and the contents of fructose and glucose were markedly higher in T449 than E249 at the single microspore stage. Our results suggested that polyploidy not only induces abrupt expression changes in the meiosis-related genes that lead to abnormal chromosome behavior, but also causes changes in the saccharide distribution and expression patterns of saccharide-related genes, which jointly causes sterility in the autotetraploid rice.
\end{abstract}

Keywords Autotetraploid rice $\cdot$ Pollen development $\cdot$ Carbohydrate metabolism $\cdot$ Saccharides' transporters $\cdot$ Meiosis

Communicated by S. Hohmann.

Lin Chen and Muhammad Qasim Shahid contributed equally to this work.

Electronic supplementary material The online version of this article (https://doi.org/10.1007/s00438-018-1471-0) contains supplementary material, which is available to authorized users.

Xiangdong Liu

xdliu@scau.edu.cn

Lin Chen

linchen163163@163.com

Muhammad Qasim Shahid

qasim@scau.edu.cn

Jinwen Wu

jwwu@scau.edu.cn

Zhixiong Chen

chenzx@scau.edu.cn

\section{Introduction}

Whole-genome duplication (WGD) or polyploidy has played an important role in plant evolution. Environmental change or stress increased the short-term adaptive potential of polyploids due to changes in gene expression patterns and increased genetic variation (Wendel 2000; Soltis et al.

\section{Lan Wang}

wanglan@scau.edu.cn

1 State Key Laboratory for Conservation and Utilization of Subtropical Agro-Bioresources, South China Agricultural University, Guangzhou 510642, China

2 Guangdong Provincial Key Laboratory of Plant Molecular Breeding, South China Agricultural University, Guangzhou 510642, China 
2009; Van de Peer et al. 2017). There are two major types of polyploids in plants: allopolyploid and autopolyploid. Allopolyploids originate through the duplication of chromosome sets of different species, a major pathway for plant evolution, which may have a survival advantage, because different chromosome sets are key determinants of adaptive success in the new environment (Soltis et al. 2009; Paun et al. 2011; Van de Peer et al. 2017). Autopolyploids involve whole-genome duplication within-species, which is more prevalent than indicated by taxonomy alone and has become an important element of plant diversity (Soltis et al. 2007; Van de Peer et al. 2017). Autopolyploid species are widely found in plants and have higher economic and resistance importance than their progenitors (Gebhardt and Valkonen 2001; Barker et al. 2016; Van de Peer et al. 2017). Autotetraploid rice has strong biological advantages over diploid rice, such as huge vegetative organs, high yield potential, and wide adaptability (Shahid et al. 2011, 2012; Tu et al. 2014; Yang et al. 2014). However, low fertility is a major obstacle in the utilization of autotetraploid rice (He et al. 2011a; Shahid et al. 2013a; Wu et al. 2015; Li et al. 2017). To improve the fertility and yield of autotetraploid rice, it is of utmost importance to investigate pollen development and its molecular mechanism in this organism.

Partial pollen sterility is a main reason for low fertility in autotetraploid rice (Shahid et al. 2010; Li et al. 2016). The previous studies have shown that abnormal chromosome behavior and abnormal microtubule organization are the main cause of pollen abortion in autotetraploid rice (He et al. 2011a, b; Wu et al. 2014). Transcriptome analysis has suggested that polyploidy enhanced multi-allelic interactions at pollen sterility loci and increased chromosomal abnormalities in autotetraploid rice (Wu et al. 2015). In addition, small RNA sequencing indicated that the partial sterilities of pollen and embryo sac were caused by specific differentially expressed miRNAs in autotetraploid rice ( $\mathrm{Li}$ et al. 2016, 2017). Two photoperiod- and thermo-sensitive genic male sterile lines (PS006 and PS012) of polyploid rice were developed and their hybrids showed great heterosis and enormous potential for the improvement of rice quality and productivity (Zhang et al. 2017).

Next-generation sequencing is widely used in genome sequencing, transcriptome sequencing, small RNA sequencing, chromatin immunoprecipitation sequencing (ChIP-Seq), and DNA methylation sequencing. Genomic re-sequence technology is an efficient way to detect genetic variations (Han and Huang 2013), which play an importance role in the QTL mapping, marker-assisted genomic selection, and haploid-type analysis (McCouch et al. 2010). Genome-wide association studies (GWAS) have concentrated on the analysis of genes associated with heading date and grain-related traits using whole-genome re-sequencing (Huang et al. 2012). Fu et al. (2016) identified numerous SNPs and InDels in two indica rice
(RGD-7S and Tai Feng B) and obtained DNA polymorphic markers by re-sequencing two rice cultivars. RNA transcript technology (RNA-seq) is a helpful tool for understanding of differentially expressed genes and the expression patterns of stage-specific genes in plants. RNA-seq has been applied to detect differentially expressed genes under abiotic stresses in diploid rice (Jin et al. 2013; Fu et al. 2017). In addition, RNAseq has also been applied to identify differentially expressed genes in autotetraploid rice compared to diploid rice during pollen development (Wu et al. 2014). Guo et al. (2017) detected many genes associated with fertility and heterosis in neo-tetraploid rice hybrids by RNA-seq. However, little information about genome-wide DNA variation combined with RNA-seq is available in autotetraploid compared to diploid rice.

To detect the genomic variations between autotetraploid rice line (T449) and its diploid rice counterpart (E249), which is a famous diploid rice variety harbouring doubleneutral genes at the $S a$ and $S b$ pollen sterility loci and the $S_{5}{ }^{n}$ gene that can overcome indica-japonica hybrid sterility (Shahid et al. 2013b) was re-sequenced using a highresolution technique. T449 had been self-crossed for more than 25 generations in our lab, and has stable agronomic traits. Moreover, chromosome behavior and histological observations were used to investigate pollen development in T449 and E249. Transcriptome analysis of anther development was also performed during meiosis and single microspore stages to detect differentially expressed genes (DEGs) between T449 and E249. This study was planned (1) to observe chromosome behavior and saccharide distribution in autotetraploid rice during pollen development and (2) to detect genes that might be associated with low pollen fertility in autotetraploid rice. We found that abrupt changes in the expression patterns of meiosis and saccharide-related genes cause low fertility in autotetraploid rice.

\section{Materials and methods}

\section{Rice material}

An autotetraploid rice line (T449) and its diploid counterpart (E249) were used in this study. T449 was developed from E249 by treatment with colchicine and self-crossed for more than 25 generations at our farm. All materials were planted at the experimental farm of South China Agricultural University (SCAU) under natural conditions, and management practices followed the recommendations for the area.

\section{Observation of chromosome behaviour and pollen fertility}

Chromosome behaviour was observed according to Wu et al. (2014). The spikelets of T449 and E249 were harvested from 
the shoots of rice plants with -2 to $4 \mathrm{~cm}$ between their flag leaf cushion and the second to last leaf cushion, and fixed in Carnoy's solution (ethanol:acetic acid $=3: 1$ ) for $24 \mathrm{~h}$. The samples were stored in $70 \%$ ethanol at $4{ }^{\circ} \mathrm{C}$ after washing. Anthers were dissected from the floret using a dissecting needle and forceps, and were squashed and placed in a small drop of $1 \%$ acetocarmine on a glass slide. After 1-2 min, the glass slide was covered with a slide cover and observed under a microscope (Motic BA200).

Pollen fertility was estimated by the ratio of normal and abnormal pollen grains, which were observed by staining with $1 \% \mathrm{I}_{2}-\mathrm{KI}$ under a microscope (Motic BA200). Normal pollens are fully stained, while abnormal pollens can be divided into two types based on staining and pollen morphology, including stained abortive pollens and empty abortive pollens. Stained abortive pollens are smaller than normal pollens or not fully stained and empty abortive pollens are smaller than normal pollens, and are empty or colourless (Zhang and Lu 1989).

\section{Histological observation}

The anthers of T449 and E249 were harvested, fixed, dehydrated, and embedded in a Leica 7022 Histeresin Embedding Kit (7022LR) according to the manufacturers' protocol (Heraeus Kulzer). Sections of 1-2 $\mu \mathrm{m}$ thickness were cut with the microtome (Leica RM2235), and were dried at $60{ }^{\circ} \mathrm{C}$ for $24 \mathrm{~h}$. The sections were stained in $0.5 \%$ periodic acid $(\mathrm{m} / \mathrm{v})$ and with Schiff reagents $[0.05 \%$ fuchsin basic $(\mathrm{m} / \mathrm{v}), 0.05 \%$ sodium metabisulphite $(\mathrm{m} / \mathrm{v})$, and $10 \% \mathrm{HCl}$ $(\mathrm{v} / \mathrm{v})$ ], and finally with $0.05 \%$ toluidine blue $(\mathrm{m} / \mathrm{v})$ (Feder and OBrien 1968). The sections of anthers were observed and photographed using a microscope (Motic BA200).

\section{Determination of saccharides' content in anther}

The anthers of T449 and E249 at the meiotic and single microspore stages were collected to confirm the pollen development stages using DAPI fluorescence staining method (Table S1). The $500 \mathrm{mg}$ samples of fresh anthers were air-dried at $110^{\circ} \mathrm{C}$ in an oven for $15 \mathrm{~min}$ and kept at $65^{\circ} \mathrm{C}$ for $12 \mathrm{~h}$ in the same oven. The $50 \mathrm{mg}$ samples of dried anthers were extracted with $80 \%$ ethanol aqueous solution, and then placed in a hot water bath at $80{ }^{\circ} \mathrm{C}$ for $40 \mathrm{~min}$. The samples were centrifuged at $4000 \mathrm{rpm}$ for $10 \mathrm{~min}$ and supernatant was removed. The same process was repeated twice and the supernatants were collected. The activated carbon was added to the supernatant for decolorization at $80^{\circ} \mathrm{C}$ for $10 \mathrm{~min}$, and the volume was adjusted to $5 \mathrm{ml}$. Finally, the mixed supernatant was filtered and prepared to measure the contents of fructose, glucose, and sucrose using kits purchased from Suzhou Keming Bioengineer Company, China. All measurements were performed in triplicate.

\section{Whole-genome re-sequencing analysis}

The genomic DNAs of E249 and T449 were extracted from young leaves using a modified CTAB method (CotaSanchez et al. 2006). The library was prepared according to the manufacturer's protocol. The genomic re-sequencing was performed on the Illumina Hiseq 2500 platform (Biomarker Technologies, Beijing, China). The procedure was performed according to the standard Illumina protocol. The generated FASTQ file quality was evaluated using FastQC (http://www.bioinformatics.babraham.ac.uk/projects/fastq c/). The low-quality reads [reads with sequencing adapter, reads with more than $10 \% \mathrm{~N}$ content, and reads with more than $50 \%$ low-quality bases $(<10)$ ] were removed, and the filtered high-quality reads were then mapped onto the Nipponbare reference genome using BWA software. The SNPs and InDels were detected by GATK software, and the base mutation of SNPs, physical location of the corresponding chromosome, the size of the small InDels, and positions on the corresponding chromosomes were detected. The copy number variations (CNVs) were identified using FREEC software, and the breakpoint threshold was 0.8 (window $=50,000$ ), while other parameters were set as default. The SNPs and InDels were annotated using SnpEff software and CNVs were annotated based on the GFF file of the Nipponbare reference genome.

\section{Validation of SNPs and InDels}

Based on the re-sequencing results, $38 \mathrm{SNPs}$ and InDels were randomly selected for validation. Primer Premier 5.0 was employed to design oligonucleotide primers, with a product length that ranged from 400 to $800 \mathrm{bp}$ (Table S2). The genomic DNAs of E249 and T449 were used as template to amplify DNA by the polymerase chain reaction (PCR). The PCR process was $94^{\circ} \mathrm{C}$ for $5 \mathrm{~min}$, followed by 35 cycles of $95{ }^{\circ} \mathrm{C}$ for $45 \mathrm{~s}, 55^{\circ} \mathrm{C}$ for $45 \mathrm{~s}$, and $72{ }^{\circ} \mathrm{C}$ for $1 \mathrm{~min}$, and a final extension at $72{ }^{\circ} \mathrm{C}$ for $5 \mathrm{~min}$. The PCR products were sequenced by Sanger sequencing, and the sequence alignments were performed using ClustalW software.

\section{RNA extraction and RNA-seq experiments}

Total RNA was extracted from anthers at the meiotic and single microspore stages. All samples were collected in three biological replicates and stored at $-80^{\circ} \mathrm{C}$ for RNA solation. The total RNA from each sample was extracted from the anthers according to the manual instructions of the TRIzol reagent (Life technologies, California, USA). The library was prepared according to the manufacturer's protocol. The RNA-seq was performed on the Illumina HiSeq 2500 sequencing platform (Biomarker Technologies, Beijing, China). Using the Illumina paired-end RNA-seq approach, 
we sequenced the transcriptome that generated millions of paired-end reads. Low-quality reads (adaptor sequences, unknown nucleotides $>5 \%$, or $Q 20<20 \%$ [percentage of sequences with sequencing error rates $<1 \%$ )] were removed. The clean reads, which were filtered from the raw reads, were mapped onto the Nipponbare reference genome using Bowtie 2 and Tophat 2 softwares with the default parameters. The aligned records from the aligners in the BAM/SAM format were further examined to remove potential duplications. Gene expression differences between samples were detected using the DESeq package (Anders and Huber 2010). The fragments per kilobase (FPKM of transcript per million fragments mapped) values were used to estimate gene expression levels (Trapnell et al. 2010). The false discovery rate (FDR) was used to determine the threshold of the $p$ value in multiple tests. The genes were used for subsequent analysis using FDR $\leq 0.05$ and the absolute value of $\log 2$ (fold change) $\geq 1$.

\section{Statistical analysis of RNA-seq data}

Hierarchical analysis was performed using Cluster 3.0 software after normalization. Venny software was used to identify the overlapped differentially expressed genes in different samples (http://bioinfogp.cnb.csic.es/tools/venny/). Transcription factor data were downloaded from the database DRTF (Jin et al. 2017). Gene ontology (GO) enrichment analysis was employed for functional categorization of differentially expressed genes using the Plant GeneSet Enrichment Analysis Toolkit (http://structuralbiology.cau. edu.cn/PlantGSEA/) and AgriGO tool (http://bioinfo.cau. edu.cn/agriGO/).

\section{Real-time qRT-PCR analysis}

A total of 18 DEGs were selected for validation by qRTPCR. The gene-specific primers were designed using Primer Premier 5.0 software (Table S3). Total RNA was taken from sequenced samples, and the first-strand cDNA was synthesized using the PrimeScript ${ }^{\mathrm{TM}}$ II first-strand cDNA synthesis kit (TaKaRa) according to the manufacturer's instructions. The qRT-PCR was performed on the Lightcycler 480 system (Roche) using the Prime Script RT reagent Kit with gDNA Eraser (TaKaRa). The qRT-PCR reaction condition was $30 \mathrm{~s}$ at $95^{\circ} \mathrm{C}$, with 40 cycles of $95{ }^{\circ} \mathrm{C}$ denaturation for $5 \mathrm{~s}$ and $60{ }^{\circ} \mathrm{C}$ annealing and extension for $20 \mathrm{~s}$. The relative expression levels of genes were calculated using the $2^{-\Delta \Delta \mathrm{Ct}}$ method (Livak and Schmittgen 2001). All qRT-PCR reactions were performed in triplicate.

\section{Data availability}

All the data were submitted to NCBI Sequence Read Archive database under accession number PRJNA436888.

\section{Results}

\section{Characterization of autotetraploid rice (T449)}

Obvious differences were detected between T449 and E249 for the main agronomic traits (Fig. 1), including plant height, grain length, grain width, 1000-grain weight, and panicle length, but there were non-significant differences in flag leaf length and width (Table 1). Grain length, grain width, and 1000-grain weight were significantly larger, and plant height and number of effective panicles were significantly lower in T449 than in E249 (Table 1 and Fig. 1). In addition, stained abortive and empty abortive pollens were found in the T449 (Fig. 1), and pollen fertility and seed setting were 54.09 and $34.47 \%$ in T449, respectively, significantly lower than E249 (Table 1). These results showed that T449 had obvious biomass advantages over its diploid counterpart, and displayed significant differences in agronomic traits.
Fig. 1 Plant type, grain shape, and pollen fertility of autotetraploid (T449) and diploid rice (E249). a Plant appearance of T449 and E249, b grains shape of T449 and E249, bar $=1 \mathrm{~cm}$. Pollen fertility of E249 (c) and T449 (d) observed by staining with $1 \% \mathrm{I}_{2}-\mathrm{KI}$. Blue arrows indicate stained abortive pollen, green arrows indicate empty abortive pollen, and red arrows indicate normal pollen. $\mathrm{Bar}=100 \mu \mathrm{m}$. (Color figure online)
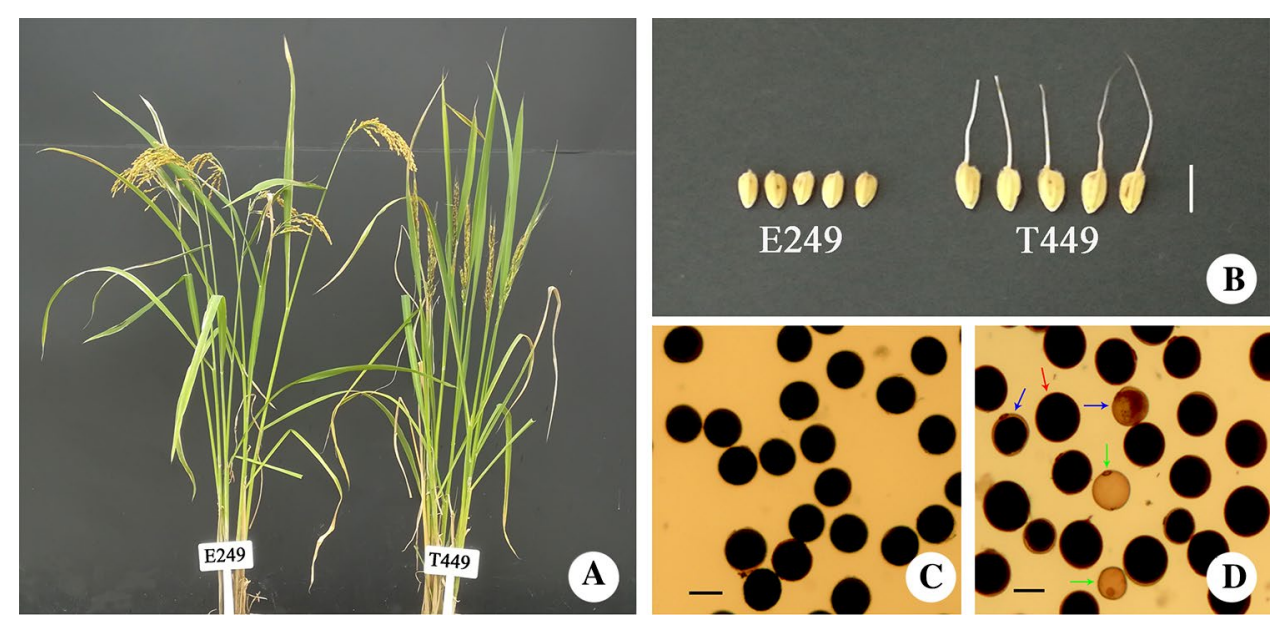
Table 1 Main agronomic traits and pollen fertility of autotetraploid rice (T449) and diploid rice (E249)

\begin{tabular}{lrc}
\hline Traits & \multicolumn{1}{l}{ E249 } & \multicolumn{1}{l}{ T449 } \\
\hline Plant height $(\mathrm{cm})$ & $96.5 \pm 2.91$ & $84.5 \pm 4.52^{* *}$ \\
Flag leaf length $(\mathrm{cm})$ & $35.36 \pm 5.83$ & $36.49 \pm 5.41$ \\
Flag leaf width $(\mathrm{cm})$ & $2.05 \pm 0.06$ & $2.04 \pm 0.11$ \\
Grain length (mm) & $7.19 \pm 0.10$ & $8.99 \pm 0.37 * *$ \\
Grain width (mm) & $3.05 \pm 0.04$ & $3.73 \pm 0.04 * *$ \\
1000-grain weight $(\mathrm{g})$ & $18.27 \pm 0.43$ & $32.34 \pm 1.37 * *$ \\
Panicle length (cm) & $23.4 \pm 1.24$ & $25.33 \pm 1.02^{* *}$ \\
Number of effective panicles & $7.35 \pm 1.27$ & $4.25 \pm 1.12^{* *}$ \\
Pollen fertility $(\%)$ & $94.43 \pm 1.72$ & $54.09 \pm 3.13 * *$ \\
Seed setting $(\%)$ & $89.38 \pm 2.33$ & $34.47 \pm 9.11^{* *}$ \\
\hline
\end{tabular}

**Significant difference at $p<0.01$

\section{Chromosome behaviour during PMC meiosis in the autotetraploid rice}

Meiotic stages in T449 were consistent with E249, and could be divided into nine development stages, including prophase I (leptotene, zygotene, pachytene, diplotene, and diakinesis) (Fig. S1 A, B and Fig. 2a-e), metaphase I (Fig. S1C and Fig. 2f), anaphase I (Fig. S1D and Fig. 2g), telophase I (Fig. S1E and Fig. 2h), prophase II, metaphase II (Fig. S1F and Fig. 2i), anaphase II (Fig. S1G and Fig. 2j), telophase II (Fig. S1H and Fig. 2k), and tetrad (Fig. S1I and Fig. 21). The bivalent was the most common chromosome configuration at diakinesis and metaphase I in E249, while tetravalent was most frequent in T449 at the same stages (Table 2). Many abnormal chromosome behaviours were observed in T449, including chromosome lagging at metaphase I (Fig. 2m), chromosome straggling at anaphase I (Fig. 2n), abnormal spindle at metaphase II (Fig. 2o), and asynchronous cell division at the triad stage (Fig. 2p). During meiosis I, the average frequencies of chromosomal abnormalities were
Fig. 2 Chromosome behaviours during PMC meiosis in an autotetraploid rice line. a Zygotene, b, c pachytene, $\mathbf{d}$ diplotene, e diakinesis, $\mathbf{f}$ metaphase, $\mathbf{i}$, $\mathbf{g}$ anaphase I, $\mathbf{h}$ telophase I, $\mathbf{i}$ metaphase II, $\mathbf{j}$ anaphase II, $\mathbf{k}$ telophase II, $\mathbf{l}$ tetrad stage, $\mathbf{m}$ abnormal metaphase I, $\mathbf{n}$ abnormal anaphase I, o abnormal metaphase II, $\mathbf{p}$ abnormal tetrad stage. $\mathrm{Bar}=10 \mu \mathrm{m}$
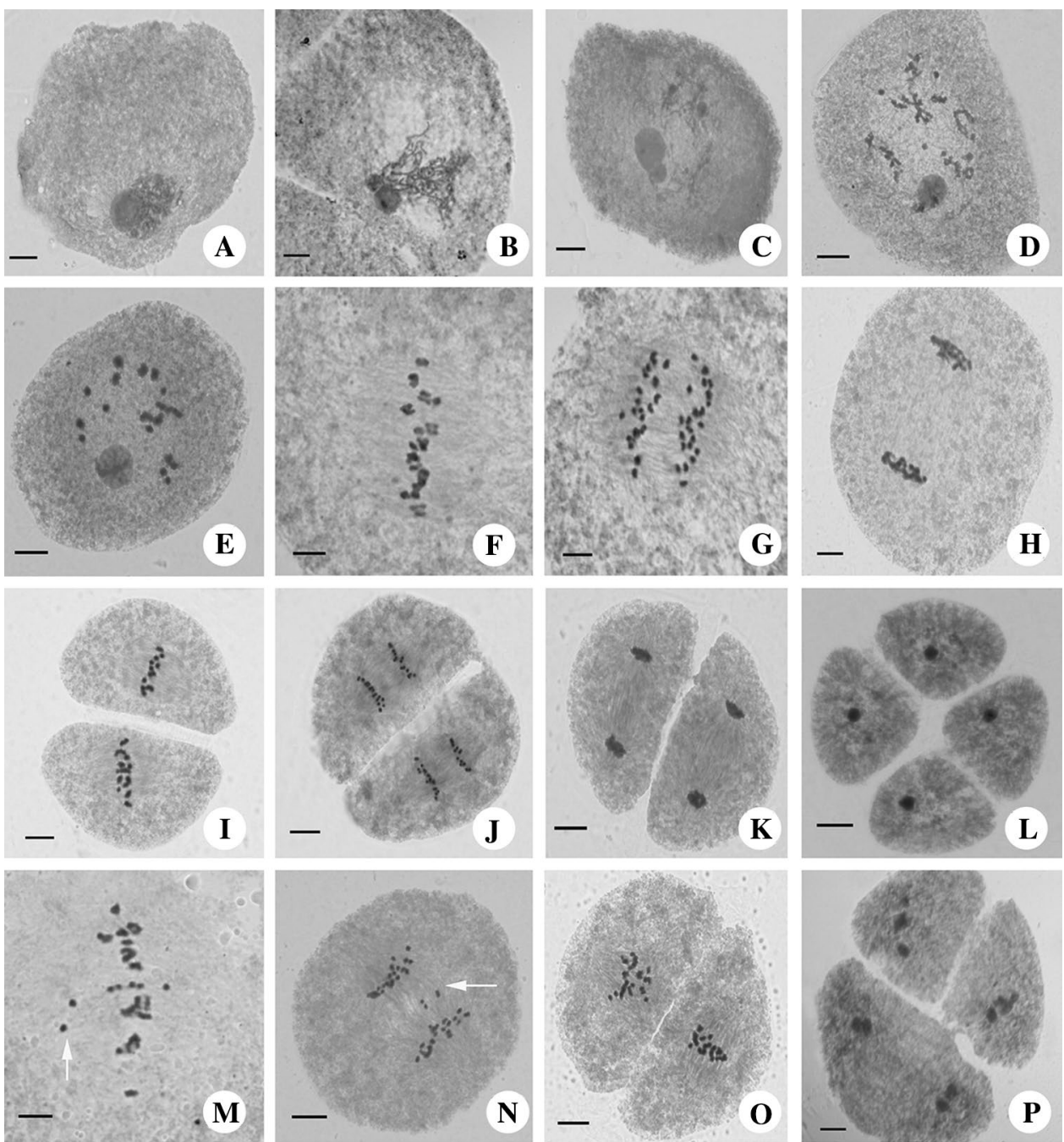
Table 2 Meiotic chromosome configurations of autotetraploid (T449) and diploid (E249) rice

\begin{tabular}{lllc}
\hline & Number of cells & Stages & Chromosome configuration \\
\hline E249 & 160 & Diakinesis & $(0.2 \pm 0.64) \mathrm{I}+(11.9 \pm 0.32) \mathrm{II}$ \\
& 159 & Metaphase I & $(0.16 \pm 0.55) \mathrm{I}+(11.92 \pm 0.27)$ \\
& & & II \\
T449 140 & Diakinesis & $(0.91 \pm 1.39) \mathrm{I}+(5.85 \pm 3.39)$ \\
& & & II $+(0.18 \pm 0.55)$ \\
& \multirow{2}{*}{142} & III $+(8.71 \pm 1.88) \mathrm{IV}$ \\
& & & $(1.29 \pm 1.6) \mathrm{I}+(6.47 \pm 3.25)$ \\
& & $\mathrm{II}+(0.2 \pm 0.58)$ \\
& & $\mathrm{III}+(8.29 \pm 1.57) \mathrm{IV}$ \\
\hline
\end{tabular}

I, II, III, and IV represent univalent, bivalent, trivalent, and tetravalent, respectively

40.36, 20.69, and 2.62\% higher in T449 than E249 at metaphase I, anaphase I, and telophase I, respectively. In meiosis II, the average abnormal frequencies were 31.62 , 56.03, 7.96, and 4.65\% higher in T449 than E249 at metaphase II, anaphase II, telophase II, and tetrad, respectively (Table 3).

\section{Whole-genome re-sequencing of autotetraploid and diploid rice}

A total of 107131936 and 100395344 clean reads were obtained in E249 and T449 using re-sequencing, and filtered from 107605398 and 100940422 raw reads, respectively. The G/C contents were 44.48 and $45.1 \%$ in E249 and T449, respectively. Approximately $98.34 \%$ (E249) and 98.13\% (T449) of clean reads were mapped onto the Nipponbare reference genome, respectively. Moreover, 94.96 and $94.44 \%$ of these reads were uniquely mapped, and 94.32 and $93.88 \%$ were found at the $10 \times$ coverage depths, and the reads' coverage depth were $37 \times$ and $35 \times$ in E249 and T449, respectively (Table 4).

To understand the genomic variations between E249 and T449, the DNA polymorphisms were further analyzed. The two filter conditions (coverage $\geq 10$ and $\leq 100$, and removal of heterozygous SNPs and InDels) were applied to minimize the detection of false-positive SNPs and InDels
Table 4 Summary of general sequencing data of autotetraploid (T449) and diploid (E249) rice mapped onto Nipponbare reference genome

\begin{tabular}{lll}
\hline & E249 & T449 \\
\hline Clean bases & 16049400548 & 15040007266 \\
Raw reads & 107605398 & 100940422 \\
Clean reads & 107131936 & 100395344 \\
Percent of bases $\geq Q 30(\%)$ & 87.31 & 86.81 \\
Mapped (\%) & 98.34 & 98.13 \\
Properly mapped (\%) & 94.96 & 94.44 \\
Coverage ratio 10× (\%) & 94.32 & 93.88 \\
GC content (\%) & 44.48 & 45.1 \\
Average coverage depth & 37 & 35 \\
\hline
\end{tabular}

between E249 and T449. A total of 81 SNPs and 182 InDels were identified in T449 compared to E249 (Table S4). To validate using Sanger sequencing, we randomly selected 38 variation sites of SNPs and InDels from E249/T449 after PCR amplification. The DNA sequences were consistent with the re-sequencing data, suggesting that the resequencing data are reliable. Only three non-synonymous SNPs and six large-effect InDels were detected, which were associated with three and six genes, respectively, including cysteine protease family gene ( $\mathrm{Os} 04 \mathrm{~g} 0402300)$, methyltransferase (Os09g0415700), pectinesterase (Os01g0634600), disease-resistance gene (Os11g0224900), verticillium wilt disease-resistance gene (Os01g0158600), two NBSLRR-type disease-resistance genes (Os11g0597700 and Os11g0686500), and two expressed genes (Os08g0528700 and $O s 10 g 0134033$, Table S4). However, there was no variation at the pollen sterility loci $S a$ and $S b$, and $S_{5}$ gene between T449 and E249. These results suggested that the reason for the low pollen fertility of T449 may not be SNP and InDel variations.

A total of $60 \mathrm{CNVs}$ were detected between E249 and T449, which were associated with 617 genes. GO-enrichment analysis of the 617 genes revealed that 13 GO terms were significantly enriched in the biological process category, and only a membrane (GO:0016020) term was
Table 3 Frequency of abnormal chromosome behaviours during meiosis in autotetraploid rice (T449) and its diploid (E249) counterpart

\begin{tabular}{lllllc}
\hline Stage & E249 & & \multicolumn{2}{l}{ T449 } \\
\cline { 2 - 3 } & Number of cells & Abnormal (\%) & & Number of cells & Abnormal (\%) \\
\hline Metaphase I & 244 & 2.05 & 275 & 40.36 \\
Anaphase I & 220 & 3.18 & 232 & 20.69 \\
Telophase I & 224 & 0.45 & 229 & 2.62 \\
Metaphase II & 256 & 3.52 & 253 & 31.62 \\
Anaphase II & 181 & 7.18 & & 141 & 56.03 \\
Telophase II & 259 & 4.63 & 289 & 7.96 \\
Tetrad stage & 249 & 1.20 & 258 & 4.65 \\
\hline
\end{tabular}


detected in the cell component category (Table S5). Five GO terms were detected in the molecular function category, including transferase activity (GO:0016740), kinase activity (GO:001630), transferase activity, transferring phosphorus-containing groups (GO:0016772), catalytic activity (GO:0003824), and lipid binding (GO:0008289). Furthermore, we compared the genes related to CNVs in T449 with the data reported for rice anther meiosis-stage-related genes (Fujita et al. 2010; Yant et al. 2013; Wright et al. 2015). We identified two meiosis-related genes from these comparisons, including OsAM1 (Os03g0650400), which plays a fundamental role in building the proper chromosome structure at the beginning of meiosis (Che et al. 2011) and RAD17 (Os03g0242100), which regulates DNA damage repair and homologous recombination (Heitzeberg et al. 2004). However, the expression patterns of two meiosis-related genes displayed non-significant changes between E249 and T449 according to qRT-PCR results (Fig. S2).

\section{Differentially expressed genes in autotetraploid and diploid rice}

Meiosis and single microspore stages play important roles in determining the pollen fertility of rice. Therefore, RNAseq was employed to study the global gene expression of the two stages in T449 compared to E249. In total, approximately 230 million clean reads were detected in anthers at the meiosis and single microspore stages. The clean reads were aligned against the Nipponbare reference genome, and 84.28-89.73\% annotated transcripts of the reference genome were obtained in T449 and E249, respectively (Table S6). All of the correlation coefficients were higher than 0.85 among the three biological replications (Table S7), and principal component analysis (PCA) showed that replicate samples of each development stage clustered together (Fig. S3), which suggests that expression patterns have high similarity between biological replications. A total of 18 genes were selected to confirm RNA-seq data using qRT-PCR, including 11 and 7 genes at the meiotic and single microspore stages, respectively. The results were consistent with the RNA-seq data (Fig. S4), suggesting that the RNA-seq data are reliable.

In total, 4944 genes displayed differential expression patterns, including 1645 and 3299 genes at the meiosis and single microspore stages in T449 compared to E249, respectively (Fig. 3a, Tables S8 and S9). Of these DEGs, 524 genes were found to be down-regulated during the meiosis stage, but up-regulated at the single microspore stage, while 336 genes were up-regulated at the meiosis stage, but down-regulated at the single microspore stage (Fig. 3b). In a comparison of the meiosis and single microspore stages, 7968 and 4659 DEGs were detected in E249 and T449, respectively. Of these DEGs, 1032 down-regulated and 1376 up-regulated genes were common in E249 and T449 (Fig.
S5A). These results showed that many genes were expressed from meiosis to single microspore stages in different ploidy rice. Comparative analysis of the meiosis and single microspore stages revealed that 280 genes were up-regulated in E249, but down-regulated in T449, and 229 genes were down-regulated in E249, but up-regulated in T449 (Fig. S5A). Some genes were specifically co-expressed in different ploidy levels and pollen development stages (Fig. S5B). These results showed that genes were transcriptionally active during the meiosis and single microspore stages in different ploidy rice. Of these 4944 DEGs, which could be divided into six groups (Fig. 3c), 160 DEGs belonged to 36 families encoding transcription factors (TFs), including 73 and 133 DEGs expressed during the meiosis and single microspore stages, respectively (Fig. S6). The NAC families were most abundant in both stages, including 12 and 16 NAC TFs at the meiotic and single microspore stages, respectively. Among them, 27 and 86 TFs were specifically expressed at the meiotic and single microspore stages, respectively. Four TFs (GATA, GRAS, MYB, and MYB-related) were significantly down-regulated, and three TFs (C3H, CO-like, and MIKC) were found up-regulated during both development stages (Table S10).

Gene ontology (GO) enrichment analysis of the 4944 DEGs showed that a total of 29 and 37 GO terms were significantly enriched at the meiosis and single microspore stages in T449 compared to E249, respectively. In the biological processes category, GO terms related to the generation of precursor metabolites and energy (GO:0006091), response to abiotic stimulus (GO:0009628), response to biotic stimulus (GO:0009607), catabolic process (GO:0009056), and carbohydrate metabolic process (GO:0005975) were detected during both development stages. In molecular function category, catalytic activity (GO:0003824) and transporter activity (GO:0005215) were enriched in both stages. A total of $11 \mathrm{GO}$ terms, such as plastid (GO:0009536), intracellular membrane-bounded organelle (GO:0043231), membranebounded organelle (GO:0043227), intracellular organelle (GO:0043229), and organelle (GO:0043226), were identified in the cell component category during both development stages (Figs. S7, S8, and S9).

KEGG pathways analysis showed that 237 of 1645 DEGs at the meiosis stage and 444 of 3299 DEGs at the single microspore stage were enriched in 26 functional terms in T449 compared to E249. Interestingly, 61 and 101 DEGs were involved in the carbohydrate metabolism categories during the meiosis and single microspore stages, respectively (Fig. S10). The aforementioned DEGs were enriched in 117 subcategories, and 28 significant terms were detected. Carbon metabolism (ko01200), glyoxylate and dicarboxylate metabolism (ko00630), glycine, serine, and threonine metabolism (ko00260), photosynthesis (ko00195), peroxisome (ko04146), photosynthesis-antenna proteins (ko00196), 
Fig. 3 Differentially expressed genes (DEGs) in autotetraploid rice compared to diploid rice at meiotic and single microspore stages. a Number of up- and down-regulated DEGs in autotetraploid rice compared to diploid counterpart during both stages. b Venn diagram of DEGs in autotetraploid rice compared to diploid rice during both stages. c Expression patterns of DEGs between autotetraploid rice and its diploid counterpart in both stages. MA and SCP represent meiosis and single microspore stages, respectively. MA-up and MAdown represent up-regulated and down-regulated genes in autotetraploid rice compared to diploid rice during meiosis stage, respectively. SCP-up and SCP-down represent up-regulated and down-regulated genes in autotetraploid rice compared to diploid rice during single microspore stage, respectively
A

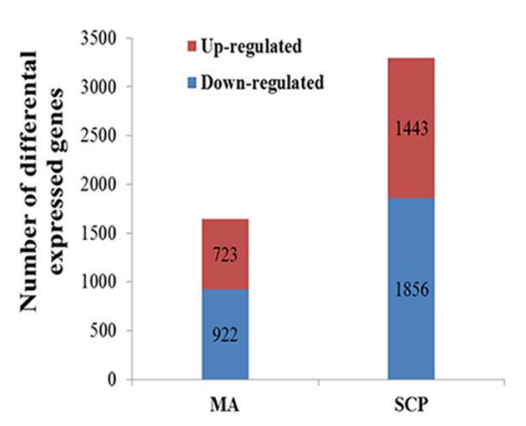

B
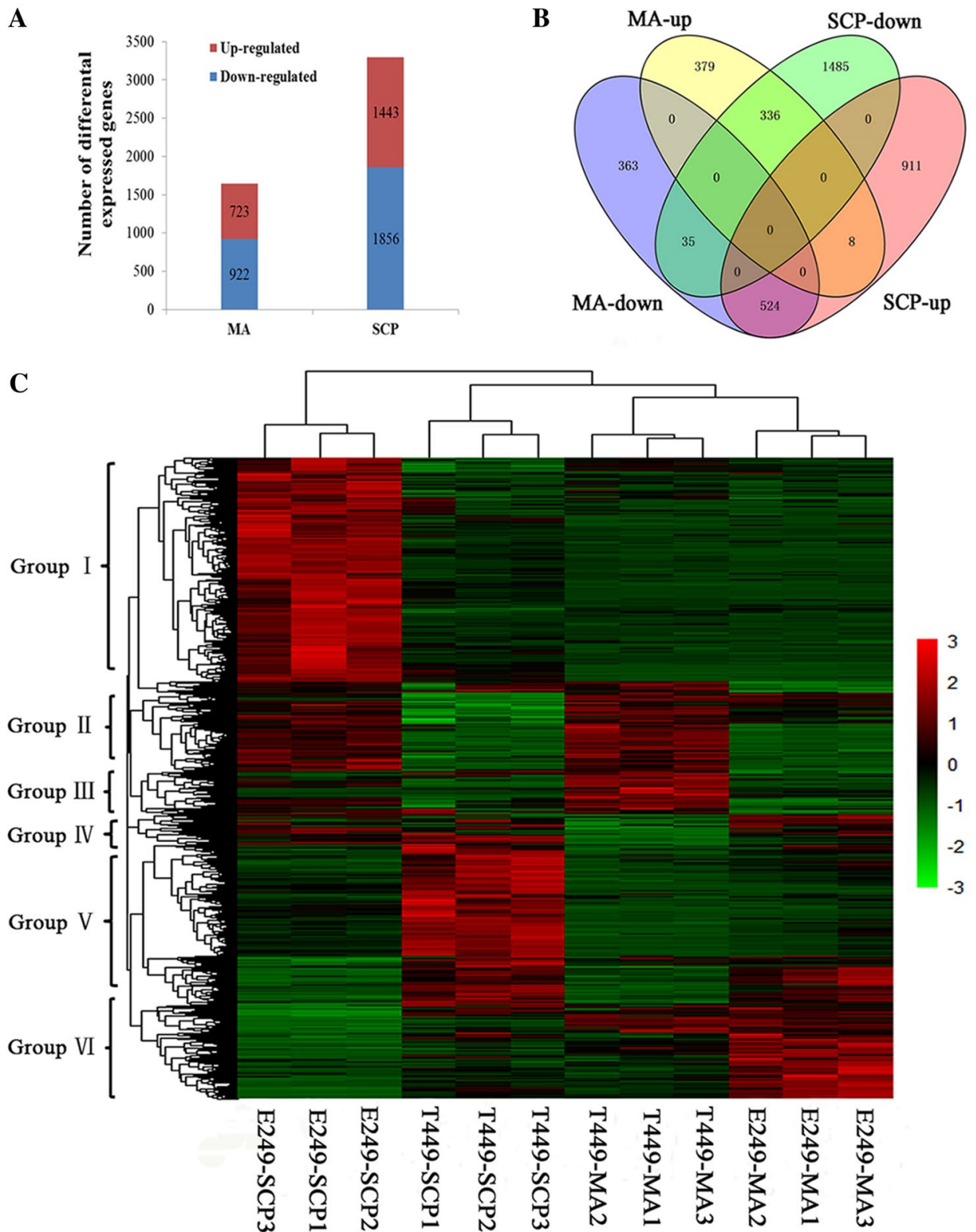

and starch and sucrose metabolism (ko00500) were significant categories during both development stages (Fig. S11).

Overall, GO and KEGG analyses results showed significant differences between T449 and E249 in the carbohydrate metabolic process during pollen development in T449 compared with E249, and DEGs were involved in sucrose synthase, glucose-6-phosphate1-dehydrogenase, 6-phosphofructokinase, and hexokinase. Sucrose is decomposed into monosaccharides by invertases and sucrose synthase, which are transported into cells and used for starch biosynthesis and other functions (Ruan et al. 2010; Ruan 2012). The sucrose synthase gene (SUS3) and hexokinase gene (OsHXK1) were significantly down-regulated at the meiosis stage. Two sucrose synthase genes (SUS2 and SUS3) and three hexokinase genes (OsHXK1,OsHXK3, and OsHXK7) were up-regulated, and a hexokinase gene (OsHXK10) was found down-regulated at the single microspore stage (Table S11). Monosaccharides and sucrose transporters play a pivotal role in saccharides' transportation from source to sink (Smeekens 2000; Wang et al. 2008). At the meiosis stage, a monosaccharide transporter (OsMST6) was found to be down-regulated. One sucrose transporter (OSSUT5) and two monosaccharide transporters (OSMST1 and OSMST8) also exhibited down-regulation at the single microspore stage, and their expression patterns were confirmed by qRTPCR (Table S11 and Fig. S4). These results indicated that saccharide transporters may cause a source-sink imbalance in T449 during pollen development. 


\section{Determination of saccharide contents in anthers during meiotic and single microspore stages}

To verify our speculation that saccharides' transporters disturb the source-sink relationship, the distribution of saccharides was investigated in T449 and E249 during pollen development. The results revealed that saccharides gathered at the connective region in E249 and T449 during the premeiotic and meiosis stages (Fig. 4). However, there was a significant difference in the distribution of saccharides between T449 and E249 during the single microspore stage (Fig. 4), and a large number of saccharides accumulated at the connective region in T449, but fewer saccharides were observed in E249 (Fig. 4). Moreover, we investigated the contents of fructose, glucose, and sucrose during pollen development. In the meiotic stage, the contents of glucose and sucrose significantly increased in T449, but there was no remarkable difference in contents of fructose between T449 and E249. At the single microspore stage, the contents of the fructose and glucose markedly increased in T449, but there was no obvious difference in the contents of sucrose between T449 and E249 (Fig. 5). These results indicated that monosaccharides (fructose and glucose) accumulated significantly in T449 during the single microspore stage.

\section{Meiosis-related and meiosis-specific genes showed down-regulation in autotetraploid rice}

As comparative transcriptome profiling was used to analyze the DEGs during pollen mother cell (PMC) meiosis, we concentrated on meiosis-stage-specific and meiosisrelated genes detected between T449 compared to E249 during the meiosis stage. We compared the DEGs to the transcriptomic data in rice or Arabidopsis meiosis-stagespecific genes and meiosis-related genes (Fujita et al. 2010; Tang et al. 2010; Deveshwar et al. 2011; Yant et al. 2013; Wright et al. 2015), and identified five meiosisrelated and 70 meiosis-stage-specific genes (Table S12).

Fig. 4 Comparison of saccharide distribution pattern in anthers of autotetraploid rice and its diploid counterpart. Anther cross sections of premeiotic stage (a), meiosis stage (b), and single microspore stage (c) in diploid rice. Anther cross sections of pre-meiotic stage (d), meiosis stage (e), and single microspore stage (f) in autotetraploid rice. $\mathrm{Bar}=100 \mu \mathrm{m}$ Arrows indicate saccharides
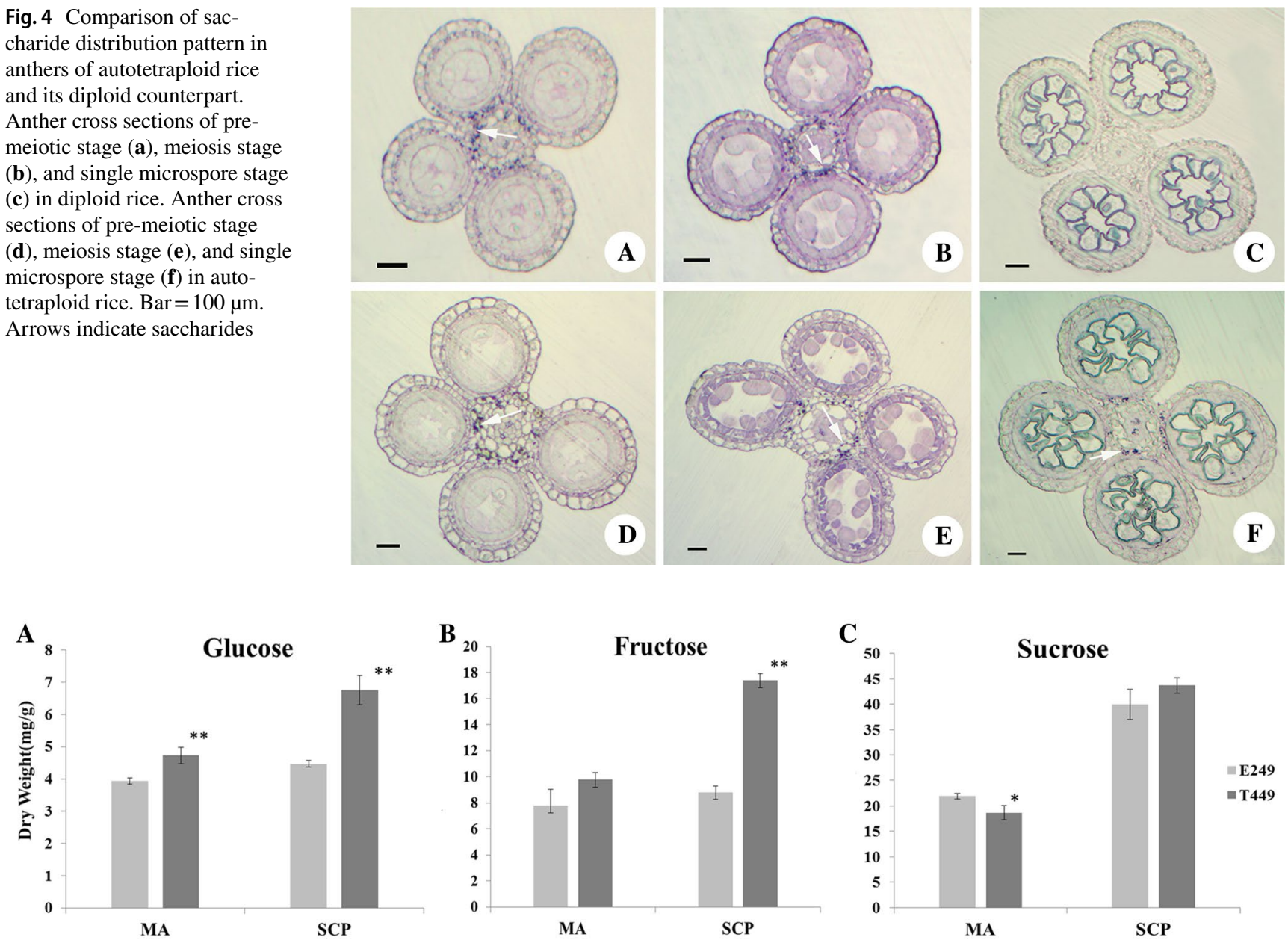

Fig. 5 Saccharides' contents in anthers of autotetraploid and diploid rice at meiotic and single microspore stages. a Glucose. b Fructose. c Sucrose. MA and SCP represent meiosis and single microspore

stages, respectively. $*$ and $* *$ represent significant difference at $p<0.05$ and $p<0.01$, respectively. (Color figure online) 
These genes exhibited at least a twofold change in T449 compared to E249, including 9 up-regulated and 66 downregulated genes. Interestingly, 60 genes were found to be down-regulated at the meiosis stage, but up-regulated at the single microspore stage, and only two genes showed the opposite tendency, i.e., up-regulated at the meiosis stage, but down-regulated at the single microspore stage. Thirteen of 75 specific genes displayed differential expression patterns at the meiosis stage, including 6 up-regulated and 7 down-regulated genes. Moreover, five meiosis-related genes were also detected, including one up-regulated and four down-regulated genes. The upregulated meiosis-related $(\mathrm{Os} 05 \mathrm{~g} 0274200)$ gene encodes DNA mismatch repair protein. Four meiosis-related genes, namely, topoisomerase B (OsMTOPVIB, Os06g0708200), F-box (OsMOF, Os04g0464966), cyclin-dependent kinase F-2 (Os10g0157200), and cyclin-dependent kinase G-1 (Os12g0432000), were found to be down-regulated, and their expression levels were verified by qRT-PCR (Fig. $\mathrm{S} 4)$. We performed the predicted protein-protein interactions of 75 meiosis-stage-specific and meiosis-related genes using STRING v10. The results showed that 17 genes constituted 2 main genetic sub-networks (Fig. 6), and all exhibited down-regulation at the meiotic stage, including 2 meiosis-related genes and 15 meiosis-specific genes. The meiosis-related gene, Os 12 g0432000, encodes cyclin-dependent kinase G-1 and interacted with two meiosis-specific genes, including two cyclin genes (Os02g0604800 and Os02g0607100). The meiosis-related gene, Os10g0157200, was annotated as cyclin-dependent kinase F-2, which interacted with five meiosis-specific genes, including two cyclin genes (OsO2g0604800 and Os02g0607100), two dimerisation domain containing genes (Skp1 family, Os09g0273800 and Os06g0113800), and a SKP1-like gene (OsO9g0274800).

\section{Discussion}

\section{Whole-genome re-sequencing reveals that low fertility was not caused by SNPs' and InDels' variations between autotetraploid and diploid rice}

Next-generation sequencing technologies have enabled re-sequencing of massive genomes, detection of DNA variations, and development of polymorphic molecular markers (Weigel and Mott 2009; Huang et al. 2012). The previous studies have reported the polymorphisms in genomic sequences of diploid rice, and discovered abundant genetic variations and important mutant genes (Jain et al. 2014; Fu et al. 2016). In the present study, a total of 82 SNPs and 182 InDels were detected in autotetraploid rice compared to diploid rice, and only three non-synonymous SNPs and six large-effect InDels were identified, which were associated with three and six genes/ proteins, respectively. These genes/proteins were related to different categories, such as four disease-resistance genes (Os11g0224900, Os01g0158600, Os11g0597700, and $O s 11 \mathrm{~g} 0686500)$, cysteine protease family genes (Os04g0402300), methyltransferase (Os09g0415700), pectinesterase (Os01g0634600), and two expressed proteins (Os08g0528700 and Os10g0134033, Table S4). This result suggests that the differences in pollen fertility between autotetraploid and diploid rice were not caused by SNP and InDel variations. Although $60 \mathrm{CNVs}$, which were associated with 617 genes, including two meiosis-related genes, were detected, the expression patterns of two meiosis-related genes exhibited non-significant differences between diploid and autotetraploid rice at the meiosis and single microspore stages by qRT-PCR. This result also revealed that $\mathrm{CNV}$ variation may not be the main reason for the low fertility of autotetraploid rice.
Fig. 6 Predicted protein-protein interaction network of meiosisspecific (black) and meiosisrelated (red) genes. Predicted protein-protein interaction subnetwork constructed using meiosis-specific and meiosisrelated differently expressed genes between autotetraploid and diploid rice during meiosis. (Color figure online)
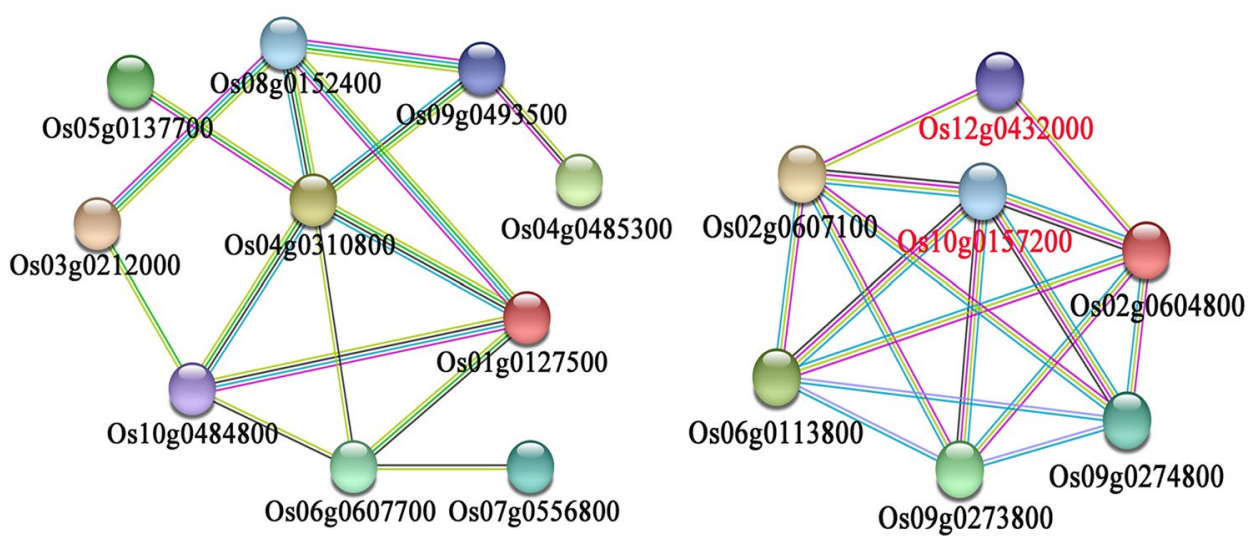


\section{Abrupt changes in meiosis-related genes and abnormal chromosome behaviour may lead to partial pollen sterility in autotetraploid rice}

Meiosis process has a great effect on plant reproductive development (Luo et al. 2014). Autotetraploid rice has four sets of homologous chromosomes, and chromosome behaviour is more complicated in autotetraploid rice than in the diploid counterpart. Abnormal chromosome behaviour is a major reason for pollen abortion in autotetraploid rice (Luan et al. 2007, 2009; He et al. 2011a, b; Wu et al. 2014). There are many types of abnormal chromosome behaviour in the autotetraploid rice, including chromosome straggling and chromosome lagging (Wu et al. 2014). In the present work, high frequency of abnormal chromosome behaviour was found in autotetraploid rice, especially in anaphase II. The chromosome configurations have obvious difference between autotetraploid rice and diploid rice, and the frequencies of univalents and multivalents were higher in autotetraploid rice than diploid rice.

In the previous studies, a number of meiosis-related genes were detected in plants (Fujita et al. 2010; Yant et al. 2013; Luo et al. 2014; Wright et al. 2015), and more than 300 meiosis-specific genes were identified in rice anther (Fujita et al. 2010; Tang et al. 2010; Deveshwar et al. 2011). The down-regulation of 1 meiosis-related and 54 meiosis-stagespecific genes increased pollen sterility loci interactions in autotetraploid rice hybrids (Wu et al. 2015). In the present research, we detected 5 meiosis-related and 70 meiosis-specific genes that showed significant differential expression patterns between autotetraploid rice and its diploid counterpart. Four meiosis-related genes displayed down-regulation, and were involved in the formation of DNA double-stranded DNA breaks (DSBs), as well as synapsis and recombination during male meiosis. OSMTOPVIB (Os06g0708200) is a TopoVIB-like protein and essential for meiotic DSB formation (Xue et al. 2016). OsMOF (Os04g0464966) is an F-box gene, which was predominantly active during leptotene to pachytene of prophase I and participates in telomere bouquet formation, homologous chromosome pairing and synapsis, and DSB repair (He et al. 2016). The down-regulation of $O S M T O P V I B$ and $O S M O F$ may increase the number of univalents and trivalents at diakinesis and metaphase I in T449. Os $10 g 0157200$ and Os12g0432000 encoded cyclindependent kinase, which is homologue to CDGK1 in Arabidopsis thaliana. CDKG1 protein kinase is essential for synapsis and recombination during male meiosis in Arabidopsis (Zheng et al. 2014). The down-regulation of $C D K G 1$ may increase the percentage of chromosomal synapsis abnormalities in autotetraploid rice. The abrupt expression patterns of these meiosis-related and meiosis-specific genes may lead to abnormal chromosome behaviour, and cause low pollen fertility in autotetraploid rice.

\section{Expression changes in saccharides' metabolism-related genes may contribute to saccharides' abnormal distribution and cause partial pollen sterility in autotetraploid rice}

The carbohydrate is the main nutrient in mature pollen that provides the necessary energy for pollen tube growth and pollen development. The meiotic and microspore stage are vital stages, which supply carbohydrates from the tapetum to microspores. This process involves the transport and degradation of polysaccharides, the formation and transport of monosaccharides, and the generation and degradation of starch (Lim et al. 2006; Kocal et al. 2008; Ruan et al. 2010). Sucrose transporters are important proteins for the translocation of sucrose from source to sink organs (Smeekens 2000). Lemoine et al. (1999) identified a pollen-specific sucrose transporter (NtSUT3) in tobacco, which participates in pollen development and supplies nutrition to pollen tubes. The deletion of the OsSUT1 gene could damage pollen function in rice (Hirose et al. 2010). These studies demonstrated that the sucrose transporter plays an important role in plant pollen development. In our study, the sucrose transporter (OsSUT5) was down-regulated in T449 during the single microspore stage, which may affect the supply of sucrose during pollen development.

Sucrose invertase and sucrose synthase are the two key enzymes in sucrose metabolism in plants (Hirose et al. 2008; Ruan 2012). Here, RNA-seq data exhibited nonsignificant changes in the expressions of sucrose invertase genes. Sucrose synthase 3 (OsSUS3) was down-regulated at the meiotic stage and up-regulated at the single microspore stage, and OsSUS2 was up-regulated at the single microspore stage. In addition, hexokinase genes have a great influence on the plant sugar signalling pathway (Cho et al. 2006, 2009; Kim et al. 2016). The hexokinase 10 (OsHXK10) plays a vital role in pollen germination, anther dehiscence, and hence grain filling in rice (Xu et al. 2008). Here, three hexokinase genes (OsHXK1,OsHXK3, and $O s H X K 7)$ were significantly up-regulated at the single microspore stage. The hexokinase genes, $O s H X K 1$ and $O s H X K 10$, were found to be down-regulated at the meiotic stage and single microspore stage in autotetraploid rice compared to the diploid counterpart. We speculated that abrupt expressions of sucrose synthase and hexokinase genes may cause abnormal pollen development in autotetraploid rice.

Monosaccharide transporters play important roles in pollen development (Wang et al. 2008). Here, cross sections of autotetraploid rice anthers showed that a number of saccharides accumulated in the connective region during the single microspore stage, unlike diploid rice (Fig. 4). Moreover, the monosaccharide contents of autotetraploid rice were significantly higher than diploid rice during single microspore stage (Fig. 5). The expression of monosaccharide transporter 8 
(OsMST8) has a pronounced effect on pollen development of rice (Mamun et al. 2006). The expression levels of OSMST8 decreased significantly in the cas mutant, which reduce the sugar contents of flower organ and cause pollen sterility (Zhang et al. 2010). Similarly, OSMST8 was found to be downregulated during the single microspore stage in autotetraploid rice, which showed partial pollen sterility. These results indicated that abnormal accumulation of monosaccharides and the differential expressions of monosaccharide transporters may lead to partial pollen abortion in autotetraploid rice.

In conclusion, abnormal chromosomal behaviour and saccharides' distribution were observed in autotetraploid rice, and many meiosis and carbohydrate metabolism-related genes displayed abrupt expression patterns during pollen development in autotetraploid rice. Therefore, we inferred that differential expression patterns of these genes may cause low pollen fertility in autotetraploid rice. Our results further the understanding of pollen development in autotetraploid rice, and revealed that carbohydrate metabolism and chromosomal behaviour abnormalities jointly cause partial pollen abortion in autotetraploid rice. Future studies will focus on the functional analysis of meiosis and saccharide metabolism-related genes, which were down-regulated in autotetraploid rice compared to diploid rice.

Acknowledgements The authors thank Ms. Shuhong Yu and other lab members for assistance.

Data availability All the data were submitted to NCBI Sequence Read Archive database under accession number PRJNA436888.

Author contributions XDL and MQS conceived and designed the experiments. LC, MQS, and XDL wrote the paper. LC, MQS, JWW, ZXC, and LW performed the experiment and analyzed the data. All authors read and approved the final manuscript.

Funding This work was supported by the National Science Foundation of China (NSFC) to XD Liu (Grant no. 31571625), Guangzhou Science and Technology Program Key Projects to XD Liu (Grant no. 201707020015), Natural Science Foundation of Guangdong Province of China to MQ Shahid (Grant no. 2017A030313142) and Guangdong Science and Technology Program to XD Liu (Grant no. 2017A030303069).

\section{Compliance with ethical standards}

Conflict of interest The authors declare that they have no competing interests.

Ethical approval This article does not contain any studies with human participants or animals performed by any of the authors.

Open Access This article is distributed under the terms of the Creative Commons Attribution 4.0 International License (http://creativeco mmons.org/licenses/by/4.0/), which permits unrestricted use, distribution, and reproduction in any medium, provided you give appropriate credit to the original author(s) and the source, provide a link to the Creative Commons license, and indicate if changes were made.

\section{References}

Anders S, Huber W (2010) Differential expression analysis for sequence count data. Genome Biol 11:R106

Barker MS, Arrigo N, Baniaga AE, Li Z, Levin DA (2016) On the relative abundance of autopolyploids and allopolyploids. New Phytol 210:391-398

Che L, Tang D, Wang K, Wang M, Zhu K, Yu H, Gu M, Cheng Z (2011) OsAM1 is required for leptotene-zygotene transition in rice. Cell Res 21:654-665

Cho Y, Yoo S, Sheen J (2006) Regulatory functions of nuclear hexokinase1 complex in glucose signaling. Cell 127:579-589

Cho JI, Ryoo N, Eom JS, Lee DW, Kim HB, Jeong SW, Lee YH, Kwon YK, Cho MH, Bhoo SH, Hahn TR, Park YI, Hwang I, Sheen J, Jeon JS (2009) Role of the rice hexokinases OsHXK5 and OsHXK6 as glucose sensors. Plant Physiol 149:745-759

Cota-Sanchez JH, Remarchuk K, Ubayasena K (2006) Ready-to-use DNA extracted with a CTAB method adapted for herbarium specimens and mucilaginous plant tissue. Plant Mol Biol Rep 24:161-167

Deveshwar P, Bovill WD, Sharma R, Able JA, Kapoor S (2011) Analysis of anther transcriptomes to identify genes contributing to meiosis and male gametophyte development in rice. BMC Plant Biol. https://doi.org/10.1186/1471-2229-11-78

Feder NED, OBrien TP (1968) Plant microtechnique: some principles and new methods. Am J Bot 55:123-139

Fu C, Liu W, Liu D, Li J, Zhu M, Liao Y, Liu Z, Zeng X, Wang F, Cloutier S (2016) Genome-wide DNA polymorphism in the indica rice varieties RGD-7S and Taifeng B as revealed by whole genome re-sequencing. Genome 59(3):197-207

Fu C, Wang F, Liu W, Liu D, Li J, Zhu M, Liao Y, Liu Z, Huang H, Zeng X, Ma X (2017) Transcriptomic analysis reveals new insights into high-temperature-dependent glume-unclosing in an elite rice male sterile line. Front Plant Sci 8:12

Fujita M, Horiuchi Y, Ueda Y et al (2010) Rice expression atlas in reproductive development. Plant Cell Physiol 51(12):2060-2081

Gebhardt C, Valkonen JP (2001) Organization of genes controlling disease resistance in the potato genome. Annu Rev Rev Phytopathol 39:79-102

Guo H, Mendrikahy JN, Xie L, Deng J, Lu Z, Wu J, Li X, Shahid MQ, Liu X (2017) Transcriptome analysis of neo-tetraploid rice reveals specific differential gene expressions associated with fertility and heterosis. Sci Rep UK. https://doi.org/10.1038/srep40139

Han B, Huang X (2013) Sequencing-based genome-wide association study in rice. Curr Opin Plant Biol 16:133-138

He J, Shahid MQ, Chen Z, Chen X, Liu X, Lu Y (2011a) Abnormal PMC microtubule distribution pattern and chromosome behavior resulted in low pollen fertility of an intersubspecific autotetraploid rice hybrid. Plant Syst Evol 291(3-4):257-265

He J, Shahid MQ, Li Y, Guo H, Cheng X, Liu X, Lu Y (2011b) Allelic interaction of $F_{1}$ pollen sterility loci and abnormal chromosome behaviour caused pollen sterility in intersubspecific autotetraploid rice hybrids. J Exp Bot 62(13):4433-4445

He Y, Wang C, Higgins JD, Yu J, Zong J, Lu P, Zhang D, Liang W (2016) MEIOTIC F-BOX is essential for male meiotic DNA double-strand break repair in rice. Plant Cell 28:1879-1893

Heitzeberg F, Chen I, Hartung F, Orel N, Angelis KJ, Puchta H (2004) The Rad17 homologue of Arabidopsis is involved in the regulation of DNA damage repair and homologous recombination. Plant J 38:954-968 
Hirose T, Scofield GN, Terao T (2008) An expression analysis profile for the entire sucrose synthase gene family in rice. Plant Sci 174:534-543

Hirose T, Zhang Z, Miyao A, Hirochika H, Ohsugi R, Terao T (2010) Disruption of a gene for rice sucrose transporter, OsSUT1, impairs pollen function but pollen maturation is unaffected. J Exp Bot 61(13):3639-3646

Huang X, Zhao Y, Wei X et al (2012) Genome-wide association study of flowering time and grain yield traits in a worldwide collection of rice germplasm. Nat Genet 44:32-39

Jain M, Moharana KC, Shankar R, Kumari R, Garg R (2014) Genome wide discovery of DNA polymorphisms in rice cultivars with contrasting drought and salinity stress response and their functional relevance. Plant Biotechnol J 12:253-264

Jin Y, Yang H, Wei Z, Ma H, Ge X (2013) Rice male development under drought stress: phenotypic changes and stage-dependent transcriptomic reprogramming. Mol Plant 6(5):1630-1645

Jin J, Tian F, Yang D, Meng Y, Kong L, Luo J, Gao G (2017) PlantTFDB 4.0: toward a central hub for transcription factors and regulatory interactions in plants. Nucleic Acids Res 45(D1):D1040-D1045

Kim H, Cho J, Ryoo N, Shin D, Park Y, Hwang Y, Lee S, An G, Jeon J (2016) Role of rice cytosolic hexokinase OsHXK7 in sugar signaling and metabolism. J Integr Plant Biol 58:127-135

Kocal N, Sonnewald U, Sonnewald S (2008) Cell wall-bound invertase limits sucrose export and is involved in symptom development and inhibition of photosynthesis during compatible interaction between tomato and Xanthomonas campestris pv vesicatoria. Plant Physiol 148:1523-1536

Lemoine R, Burkle L, Barker L, Sakr S, Kuhn C, Regnacq M, Gaillard C, Delrot S, Frommer WB (1999) Identification of a pollenspecific sucrose transporter-like protein NtSUT3 from tobacco. FEBS Lett 454(3):325-330

Li X, Shahid MQ, Wu J, Wang L, Liu X, Lu Y (2016) Comparative small RNA analysis of pollen development in autotetraploid and diploid rice. Int J Mol Sci 17(4):499

Li X, Shahid MQ, Xia J, Lu Z, Fang N, Wang L, Wu J, Chen Z, Liu X (2017) Analysis of small RNAs revealed differential expressions during pollen and embryo sac development in autotetraploid rice. BMC Genom 18:129

Lim JD, Cho JI, Park YI, Hahn TR, Choi SB, Jeon JS (2006) Sucrose transport from source to sink seeds in rice. Physiol Plant 126:572-584

Livak KJ, Schmittgen TD (2001) Analysis of relative gene expression data using real-time quantitative PCR and the 2-DELTADELTACT method. Methods 25:402-408

Luan L, Tu SB, Long WB, Wang X, Liu YH, Kong FL, He T, Yan WG, $\mathrm{Yu}$ MQ (2007) Cytogenetic studies on two $\mathrm{F}_{1}$ hybrids of autotetraploid rice varieties showing extremely high level of heterosis. Plant Syst Evol 267:205-213

Luan L, Wang X, Long WB, Liu YH, Tu SB, Xiao XY, Kong FL (2009) A comparative cytogenetic study of the rice (Oryza sativa L.) autotetraploid restorers and hybrids. Genetika 45(9):1225-1233

Luo Q, Li Y, Shen Y, Cheng Z (2014) Ten years of gene discovery for meiotic event control in rice. J Genet Genom 41:125-137

Mamun EA, Alfred S, Cantrill LC, Overall RL, Sutton BG (2006) Effects of chilling on male gametophyte development in rice. Cell Biol Int 30:583-591

McCouch SR, Zhao K, Wright M, Tung C, Ebana K, Thomson M, Reynolds A, Wang D, DeClerck G, Ali ML, McClung A, Eizenga G, Bustamante C (2010) Development of genome-wide SNP assays for rice. Breed Sci 60:524-535

Paun O, Bateman RM, Fay MF, Luna JA, Moat J, Hedren M, Chase MW (2011) Altered gene expression and ecological divergence in sibling allopolyploids of Dactylorhiza (Orchidaceae). BMC Evol Biol 11:113

Ruan Y (2012) Signaling role of sucrose metabolism in development. Mol Plant 5:763-765

Ruan Y, Jin Y, Yang Y, Li G, Boyer JS (2010) Sugar input, metabolism, and signaling mediated by invertase: roles in development, yield potential, and response to drought and heat. Mol Plant 3:942-955

Shahid MQ, Sun J, Wei C, Zhang P, Liu X (2010) Study on the abnormality of embryo sac and pollen fertility in autotetraploid rice during different growing seasons. Pak J Bot 42:7-19

Shahid MQ, Liu G, Li J, Naeem M, Liu X (2011) Heterosis and gene action study of agronomic traits in diploid and autotetraploid rice. Acta Agric Scand Sect B Soil Plant Sci 61:23-32

Shahid MQ, Xu H, Lin S, Chen Z, Naeem M, Li Y, Liu X (2012) Genetic analysis and hybrid vigor study of grain yield and other quantitative traits in autotetraploid rice. Pak J Bot 44:237-246

Shahid MQ, Li Y, Saleem MF, Naeem M, Wei C, Liu X (2013a) Yield and yield components in autotetraploid and diploid rice genotypes (indica and japonica) sown in early and late seasons. Aust J Crop Sci 7:632

Shahid MQ, Chen F, Li H, Wang S, Chen P, Lin S, Liu X, Lu Y (2013b) Double-neutral genes, $S a-n$ and $S b-n$, for pollen fertility in rice to overcome indica $\times$ japonica hybrid sterility. Crop Sci 53:164-176

Smeekens S (2000) Sugar-induced signal transduction in plants. Annu Rev Plant Physiol Plant Mol Biol 51:49-81

Soltis DE, Soltis PS, Schemske DW, Hancock JF, Thompson JN, Husband BC, Judd WS (2007) Autopolyploidy in angiosperms: have we grossly underestimated the number of species? Taxon $56: 13-30$

Soltis DE, Albert VA, Leebens-Mack J, Bell CD, Paterson AH, Zheng C, Sankoff D, DePamphilis CW, Wall PK, Soltis PS (2009) Polyploidy and angiosperm diversification. Am J Bot 96:336-348

Tang X, Zhang Z, Zhang W, Zhao X, Li X, Zhang D, Liu Q, Tang W (2010) Global gene profiling of laser-captured pollen mother cells indicates molecular pathways and gene subfamilies involved in rice meiosis. Plant Physiol 154:1855-1870

Trapnell C, Williams BA, Pertea G, Mortazavi A, Kwan G, van Baren MJ, Salzberg SL, Wold BJ, Pachter L (2010) Transcript assembly and quantification by RNA-Seq reveals unannotated transcripts and isoform switching during cell differentiation. Nat Biotechnol 28:174-511

Tu Y, Jiang A, Gan L, Hossain M, Zhang J, Peng B, Xiong Y, Song Z, Cai D, Xu W, Zhang J, He Y (2014) Genome duplication improves rice root resistance to salt stress. Rice 7:15

Van de Peer Y, Mizrachi E, Marchal K (2017) The evolutionary significance of polyploidy. Nat Rev Genet 18:411-424

Wang Y, Xiao Y, Zhang Y, Chai C, Wei G, Wei X, Xu H, Wang M, Ouwerkerk PBF, Zhu Z (2008) Molecular cloning, functional characterization and expression analysis of a novel monosaccharide transporter gene OsMST6 from rice (Oryza sativa L.). Planta 228:525-535

Weigel D, Mott R (2009) The 1001 genomes project for Arabidopsis thaliana. Genome Biol 10:107. https://doi.org/10.1186/ gb-2009-10-5-107

Wendel JF (2000) Genome evolution in polyploids. Plant Mol Biol 42:225-249

Wright KM, Arnold B, Xue K, Surinova M, O'Connell J, Bomblies K (2015) Selection on meiosis genes in diploid and tetraploid Arabidopsis arenosa. Mol Biol Evol 32:944-955

Wu J, Shahid MQ, Guo H, Yin W, Chen Z, Wang L, Liu X, Lu Y (2014) Comparative cytological and transcriptomic analysis of pollen development in autotetraploid and diploid rice. Plant Reprod 27:181-196

Wu J, Shahid MQ, Chen L, Chen Z, Wang L, Liu X, Lu Y (2015) Polyploidy enhances $F_{1}$ pollen sterility loci interactions that increase 
meiosis abnormalities and pollen sterility in autotetraploid rice. Plant Physiol 169:2700-27171

Xu F, Li X, Ruan Y (2008) RNAi-mediated suppression of hexokinase gene OsHXK10 in rice leads to non-dehiscent anther and reduction of pollen germination. Plant Sci 175:674-684

Xue Z, Li Y, Zhang L, Shi W, Zhang C, Feng M, Zhang F, Tang D, Yu H, Gu M, Cheng Z (2016) OsMTOPVIB promotes meiotic DNA double-strand break formation in rice. Mol Plant 9:1535-1538

Yang PM, Huang QC, Qin GY, Zhao SP, Zhou JG (2014) Different drought-stress responses in photosynthesis and reactive oxygen metabolism between autotetraploid and diploid rice. Photosynthetica 52:193-202

Yant L, Hollister JD, Wright KM, Arnold BJ, Higgins JD, Franklin FCH, Bomblies K (2013) Meiotic adaptation to genome duplication in Arabidopsis arenosa. Curr Biol 23:2151-2156
Zhang G, Lu Y (1989) Genetic studies of the hybrid sterility in cultivated rice (Oryza sativa). I. Diallel analysis of the hybrid sterility among isogenic $\mathrm{F}_{1}$ sterile lines. Chin J Rice Sci 3:97-100

Zhang H, Liang W, Yang X, Luo X, Jiang N, Ma H, Zhang D (2010) Carbon starved anther encodes a MYB domain protein that regulates sugar partitioning required for rice pollen development. Plant Cell 22:672-689

Zhang X, Zuo B, Song Z, Wang W, He Y, Liu Y, Cai D (2017) Breeding and study of two new photoperiod- and thermo-sensitive genic male sterile lines of polyploid rice (Oryza sativa L.). Sci Rep UK. https://doi.org/10.1038/s41598-017-15241-8

Zheng T, Nibau C, Phillips DW, Jenkins G, Armstrong SJ, Doonan JH (2014) CDKG1 protein kinase is essential for synapsis and male meiosis at high ambient temperature in Arabidopsis thaliana. Proc Natl Acad Sci 111:2182-2187 